\title{
RAC - Rivista di Antropologia Contemporanea
}

il Mulino, Bologna, 2020

\section{Pietro Meloni}

\section{CpenEdition}

\section{Journals}

Edizione digitale

URL: https://journals.openedition.org/aam/4083

DOI: $10.4000 /$ aam. 4083

ISSN: 2038-3215

\section{Editore}

Dipartimento Culture e Società - Università di Palermo

Notizia bibliografica digitale

Pietro Meloni, «RAC - Rivista di Antropologia Contemporanea», Archivio antropologico mediterraneo [Online], Anno XXIV, n. 23 (1) | 2021, online dal 30 juin 2021, consultato il 02 juillet 2021. URL: http:// journals.openedition.org/aam/4083 ; DOI: https://doi.org/10.4000/aam.4083

Questo documento è stato generato automaticamente il 2 juillet 2021.

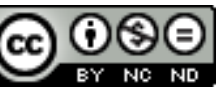

Archivio antropologico mediterraneo è distribuita con Licenza Creative Commons Attribuzione - Non commerciale - Non opere derivate 4.0 Internazionale. 


\section{RAC - Rivista di Antropologia Contemporanea}

il Mulino, Bologna, 2020

Pietro Meloni

NOTIZIA

RAC - Rivista di Antropologia Contemporanea, il Mulino, Bologna, 2020.

ISSN 2724-3168 
Nel dicembre 2020 è uscito il primo numero di una nuova rivista per il settore scientifico M-DEA/01, RAC - Rivista di Antropologia Contemporanea, edita da il Mulino e fondata da Fabio Dei. Se escludiamo il breve passaggio de L'Uomo, RAC è la prima rivista pienamente antropologica a entrare nel catalogo de il Mulino - Studi Culturali ed Etnografia e Ricerca Qualitativa, riconosciute nel settore, nascono infatti in ambiente sociologico. RAC, nelle parole del direttore, si propone "di unire scuole e indirizzi di studio diversi, portando elementi di unità e compattezza nel dibattito scientifico». Il contemporaneo della rivista è da leggersi dunque come desiderio di confronto con i grandi dibattiti internazionali che attraversano il mondo globale e la disciplina antropologica. Questa scelta rispecchia lo stile del direttore, attento alle scuole di pensiero, all'analisi teorica e alla discussione delle ricerche etnografiche, con una vocazione alla critica culturale e all'eterogeneità dei punti di vista. Contemporaneo, in questo senso, sembra voler dire "che ci riguarda tutti" e, al contempo, portare al centro del dibattito italiano temi talvolta poco frequentati, di nicchia oppure che velocemente sono assunti a paradigmi dominanti della disciplina. Contemporaneo, infine, è anche privilegiare un dialogo fatto di frizioni e di disaccordi, riprendendo, in qualche modo, una linea che il direttore Dei aveva in passato sperimentato con la rivista Ossimori.

RAC ha carattere monografico e privilegia il dibattito teorico e la ricerca etnografica. Il primo numero, che qui presento, è dedicato alla svolta ontologica, il secondo, appena uscito e curato da Matteo Aria, riguarda il tema dell'occulto, del segreto e dell'indicibile; il terzo, che uscirà alla fine del 2021 a cura di Piero Vereni, ha al centro della riflessione il potere, la sovranità e lo Stato mentre, uno dei numeri successivi, a cura di Angela Biscaldi e Vincenzo Matera, sarà dedicato ai social media.

Il numero 1 di RAC si confronta con uno dei temi probabilmente più rilevanti del dibattito antropologico di quest'ultimo decennio, quello relativo alla svolta ontologica con contributi di Antonino Colajanni, Alessandro Mancuso, Federico Scarpelli e Carlo Severi. Il fascicolo accoglie inoltre una sezione dedicata alla pandemia da Covid-19, di cui dirò.

Il saggio di Colajanni, Il 'prospettivismo' e le 'ontologie' indigene amerindiane. Una ricostruzione storico-critica del dibattito degli ultimi decenni che apre il volume, ricostruisce, in modo minuzioso e attento, il dibattito sulle ontologie amerindie a partire dalla nozione di prospettivismo. Colajanni ci mostra come questo concetto, attribuito a Viveiros de Castro, risalga ad alcuni anni prima rispetto al noto saggio "Images of Nature and Society in Amazonian Ethnology" apparso sull'Annual Review of Anthropology, precisamente al 1990 con il saggio “Ecosofia Makuna” di Kay Arhem.

Archivio antropologico mediterraneo, Anno XXIV, n. 23 (1) | 2021 
5 La "qualità prospettica" che definisce i principi della svolta ontologica, deriva da altre due espressioni: il multinaturalismo e il punto di vista. Se il multinaturalismo serve a Viveiros de Castro per contrapporsi al multiculturalismo occidentale, negando così l'idea di un'unica natura in una molteplicità di culture, la nozione di punto di vista che per quanto possa somigliare al relativismo e al costruttivismo, secondo l'antropologo brasiliano va distinta da questi altri due termini - rimanda a un mondo che esiste per qualcuno, implicando una realtà possibile solo in relazione al soggetto. La relazione con i processi di soggettivazione che molto influenza Viveiros de Castro, è ereditata dal poststrutturalismo francese, in particolare dai filosofi Gilles Deleuze e Félix Guattari - non a caso, nell'introduzione di Metafisiche Cannibali, Viveiros de Castro confessa di aver pensato a un libro non scritto il cui titolo, l'anti-Narciso, richiama esplicitamente l'anti-Edipo dei due filosofi francesi, come ben evidenzia anche Roberto Beneduce nella postfazione all'edizione italiana del libro. Bruno Latour, in particolare quello di Non siamo mai stati moderni è un altro riferimento chiave di Viveiros de Castro. Latour, come osserva Colajanni, serve all'antropologo brasiliano per confermare l'importanza di "prendere sul serio il pensiero nativo".

6 Colajanni nutre forti dubbi sull'unità delle popolazioni amerindie così come vengono presentate negli autori della svolta ontologica. Inoltre nota come il dibattito sia aumentato a seguito della pubblicazione di Oltre natura e cultura di Philippe Descola che in Italia arriva in traduzione grazie a Nadia Breda che ne ha curato le due edizioni, del 2014 e del 2021 - che, pur avanzando diverse critiche al prospettivismo, lo include nella sua trattazione in modo assai rilevante. Il libro di Descola, sempre secondo Colajanni, ha l'effetto di portare Viveiros de Castro al centro di un dibattito contemporaneo internazionale aumentandone così la rilevanza e il favore dentro la disciplina antropologica - e non solo. Il grande successo della svolta ontologica è accompagnato anche da una mancanza di critiche. In Brasile, dice Colajanni, soltanto Alcida Rita Ramos, Luiz Costa e Carlos Fausto hanno riservato critiche al lavoro di Viveiros de Castro.

7 In conclusione del suo saggio, Colajanni vede nel prospettivismo e nella svolta ontologica delle parole chiave di una disciplina - l'antropologia - che, da Writing Culture in poi, manifesta il bisogno di una continua innovazione accompagnata da una certa difficoltà al confronto. Viveiros de Castro, secondo Colajanni, fa parte di quegli studiosi poco inclini al confronto con i colleghi, una ritrosia alimentata anche da una scrittura molto spesso di difficile accesso. Il continuo ricorso alla filosofia, inoltre, sembrerebbe mettere in secondo piano l'etnografia, talvolta sottomessa al più ampio discorso teorico. I meriti, pochi secondo Colajanni, sono relativi all'aver portato le concezioni indigene al centro di un dibattito internazionale.

8 Se il saggio di Colajanni ricostruisce il dibattito sudamericano, quello di Mancuso, La 'svolta ontologica' e le questioni epistemologiche in antropologia, rende conto degli autori che lo hanno introdotto in Italia - tra gli antropologi Mara Benadusi, Domenico Branca, Nadia Breda, Valentina Gamberi, Alessandro Lutri, Francesco Remotti, oltre allo stesso Mancuso. Se l'apporto principale della svolta ontologica è stato quello di riconoscere che la dicotomia natura/cultura non è presente ovunque, un effetto secondario - ma non meno importante - è stato quello di fornire un nuovo impulso a dialogare con altre discipline - la filosofia, la biologia, l'antropologia fisica e le scienze naturali. Qui non solo gli antropologi della svolta ontologica ma anche altri autori che, per certi versi, possono essere considerati affini - Tim Ingold, Anna Tsing, Donna Haraway solo per 
citarne alcuni - hanno accolto la sfida di portare l'antropologia oltre i propri confini disciplinari, rimettendo in discussione ulteriori dicotomie: mente/corpo, organismo/ persona, universalismo/relativismo.

Per Mancuso l'intenzione di Viveiros de Castro è di superare la "crisi della rappresentazione" generata dall'antropologia postmoderna. Mancuso riprende il centro della critica di Viveiros de Castro, ovvero la rimessa in discussione della propria epistemologia, che non può più essere privilegiata rispetto alle epistemologie delle altre popolazioni. Un'epistemologia "politica", come ha scritto Beneduce nella postfazione di Metafisiche cannibali, che ci impone di riconoscere le asimmetrie di valore tra l'epistemologia dell'antropologo e quella della popolazione oggetto di indagine. Così, la dicotomia natura/cultura, per lungo tempo universalizzata, è un esempio di questa asimmetria. Martin Holbraad e Morten Pedersen, curatori del rilevante volume del 2017 The Ontological Turn. An Anthropological Exposition, sostengono che anche le posizioni relativiste e costruttiviste siano il prodotto di costruzioni culturali e, dunque, ancorate al concetto di cultura in opposizione a quello di natura. La svolta ontologica, nella sua critica epistemologica, può dunque essere vista come un tentativo di decolonizzare la disciplina dall'immaginario occidentale - che, peraltro, l'ha prodotta.

Prendere sul serio le filosofie native, dunque, vuol dire ripensare anche l'esperienza etnografica, che diventa una sperimentazione "con" l'altro, in modo simile a quell'idea di antropologia che Ingold ha portato avanti nei suoi ultimi lavori. Prendere sul serio i concetti indigeni, dice Mancuso, vuole dire considerarli "idee filosofiche che costruiscono mondi possibili”. L'etnografia, parafrasando Holbraad, può trasformarsi in ontografia, una nuova veste epistemologica che si pone come obiettivo di eliminare qualunque asimmetria tra le idee e le epistemologie del ricercatore e quelle degli "indigeni". Questo significa anche evitare di interpretare e decifrare i concetti nativi per vederli nei loro propri termini. Mancuso, dunque, ricostruisce il dibattito sulla svolta ontologica mettendo in luce questioni politiche ed epistemologiche, passando tra diversi autori collegati tra loro, quali Viveiros de Castro, Descola, Latour, Holbraad il cui contributo principale è stato di rimettere in discussione il "fondamentalismo ontologico dell'Occidente". Certo, non manca di notare come spesso questi autori siano più attenti alla promozione del loro approccio, piuttosto che dialogare con altri studi che, similmente, hanno cercato di riflettere sui problemi della rappresentazione, dell'epistemologia e della politica - tra i tanti, ad esempio, Clifford Geertz e Michel Foucault.

11 Il saggio di Scarpelli, L'antropologia culturale e le due ontologie, riporta il dibattito in una sorta di luogo originario, la filosofia, discutendo in modo critico i lavori di Viveiros de Castro in relazione all'ontologia sociale di John Searle. Scarpelli evidenzia come l'ontologia di Searle muova in direzione opposta a quella di Viveiros de Castro, tanto da parlare di due ontologie: una che critica la rappresentazione, l'altra che vuole riaffermarne la centralità, una che vuole liberarsi del naturalismo, l'altra che vuole estenderne la validità. L'ontologia sociale si sviluppa intorno alla nozione di intenzionalità, da intendersi come le modalità con cui la mente entra in relazione con il mondo esterno. Benché Holbraad e Pedersen sostengano che la svolta ontologica si sia sviluppata indipendentemente dall'ontologia sociale di Searle, Scarpelli rintraccia alcune relazioni importanti. Latour, ad esempio, si confronta con Searle sulla distinzione tra fatti dipendenti e indipendenti dalle rappresentazioni. Anche Viveiros de Castro, nelle conferenze di Cambridge richiama l'ontologia sociale, in questo caso 
come qualcosa da superare, rendendo subito evidente quella prospettiva West vs the rest che Scarpelli individua come uno dei limiti maggiori del prospettivismo.

Nella seconda generazione della svolta ontologica, quella che fa capo a Holbraad e Pedersen, gli aspetti metodologici prendono il sopravvento sull'ideologia politica - che era invece il cuore della proposta di Viveiros de Castro. Questi autori vedono la sperimentazione concettuale della svolta ontologica come qualcosa di più affine alla filosofia che all'antropologia. Il risultato è un ulteriore allontanamento dall'antropologia come disciplina empirica. Scarpelli ben comprende il successo e il fascino di una corrente del genere, capace di sfruttare una crisi disciplinare i cui problemi sono di natura politica, epistemologica, teorico-critica. Nutre però dei forti dubbi su una facile applicabilità dell'ontologia postulata da Viveiros de Castro a un'antropologia del noi. Se, come ci dice, è vero che non prendere posizione in antropologia sia spesso un elemento di aiuto, il problema dell'attendibilità - della ricerca, delle fonti, della restituzione - non può essere eluso come irrilevante o d'intralcio.

Il saggio di Severi, La trasmutazione degli esseri. Proposta per un'antropologia del pensiero, chiude la prima sezione. Si tratta, in questo caso, della traduzione di un saggio apparso nel 2014 nella rivista HAU, Transmutating beings. A proposal for an anthropology of thought, inserito perché porta una critica alla svolta ontologica a partire da una solida ricerca di campo. Benché Severi dialoghi - per la sua formazione disciplinare e per i suoi campi di ricerca - con gli autori della svolta ontologica, il suo approccio, che intreccia antropologia cognitiva, dell'arte e del pensiero, si discosta molto da questi stessi autori. Il suo articolo discute il tema della trasmutazione presso alcune popolazioni amerindie - Wayana, Yekwana, Wayampi - con l'intento di dare forma a un'antropologia del pensiero in contrapposizione alla svolta ontologica. Severi, infatti, ritiene che l'ontologia di cui parlano molti antropologi, sia piuttosto una "filosofia naturale priva di ontologia". Le cosmologie melanesiane e amazzoniche non sono, a suo avviso, né ontologie né metafisiche indigene. Pensarle come tali significa renderle degli artefatti antropologici. Gli esempi etnografici che riporta sulla trasmutazione degli esseri, permettono di rilevare l'esistenza di un'ontologia plurale, diversificata, che propone un diverso ordine degli esseri. L'idea di Severi, in opposizione a quella di Viveiros de Castro, è che un concetto come quello di ontologia deve riferirsi a diverse forme di pensiero e non, invece, a concezioni del mondo legate a diversi linguaggi.

14 I quattro autori presentano un quadro sull'ontologia che dialoga principalmente con Viveiros de Castro e, in maniera minore, con Holbraad e Pedersen. Stranamente Descola è soltanto evocato, Roy Wagner quasi inesistente, così come Eduardo Kohn e Arturo Escobar, richiamati velocemente nei diversi articoli. Certo, gli articoli nel loro insieme restituiscono un quadro molto approfondito del dibattito, anche se appare un po' schiacciato sulla figura di Viveiros de Castro.

Nell'insieme emerge un quadro ricco di spunti e interrogativi. L'opacità di alcuni concetti e l'ambiguità di alcune posizioni erano state evidenziate anche da Descola, che in Oltre natura e cultura scrive come Viveiros de Castro, pur opponendosi alla tesi sociocentrica del corpo sviluppata da Durkheim, la riproduca in parte quando riprende Leibniz sul punto di vista delle monadi. È di particolare importanza notare anche quanto la svolta ontologica condivida degli aspetti con gli studi di cultura materiale basti pensare, su questo, alla produzione scientifica di Latour, che approda a una riflessione sulla natura come soggetto di diritto passando per gli studi sugli oggetti e la 
teoria dell'actor-network theory. Pur rifiutando, come affermano gli autori presenti nel volume, un confronto con altri orientamenti teorici, la svolta ontologica sembra essere un interessante oggetto di riflessione, che ci invita a mettere in dubbio - o riprendere in considerazione - le nostre certezze.

La seconda parte del volume, sulla quale per economia di spazio mi soffermerò più brevemente, è relativa alla pandemia da Covid-19 che da oltre un anno ci riguarda tutti. Apparentemente distante dalla svolta ontologica, gli articoli sul Covid-19 offrono la possibilità di ampliare il discorso sui punti di vista, sul ripensamento dell'antropologia e sul rapporto con i non-umani.

L'articolo che apre la sezione, E se gli antropologi avessero qualcosa di intelligente (e di utile) da dire sul Covid-19? di Giuseppe Scandurra, mentre ripercorre la produzione antropologica sull'argomento - sorprendentemente vasta in così poco tempo - si chiede cosa possa dire l'antropologia sul fenomeno pandemico. Perché il Covid-19 ha certamente sollevato domande complesse e rimesso in discussione le certezze di tutti. Gli antropologi hanno dovuto fare i conti con l'impossibilità di praticare l'etnografia, strumento di indagine privilegiato e che, in qualche modo, rappresenta il tratto distintivo della disciplina. Hanno riflettuto su come ripensare il campo etnografico quando il campo ci è precluso, recuperando metodi di nicchia come la netnografia, l'autoetnografia, l'etnografia digitale, l'etnografia retrospettiva. Insieme a questi metodi hanno rifatto la loro comparsa i questionari, le interviste telefoniche e le video interviste. In molti casi è sembrata una soluzione di comodo, un modo per continuare a fare antropologia; per vedere se questi metodi verranno adottati in modo sistematico bisognerà aspettare del tempo.

Piero Vereni, nel saggio A scuola dal virus? Pandemia e doppi legami del sistema educativo, sperimenta l'autoetnografia - insieme a una più classica raccolta di dati - in relazione all'esperienza della didattica a distanza durante la pandemia. Il saggio di Julio Cesar Adiala, Cosa sta succedendo in Brasile? Il virus e il dilemma nazionale, è una lettura della condizione brasiliana durante la prima fase della pandemia, vista da un brasiliano in Italia. Qui il virus è letto nella sua valenza sociale e politica, come elemento di crisi. Il virus, dice Adiala, non è solo un fenomeno naturale ma è anche un costrutto sociale, $\mathrm{i}$ cui significati sono da leggersi negli specifici interessi culturali e politici. Così, in Brasile, la pandemia è un modo per leggere le tensioni politiche che attraversano il paese.

Il saggio di Antonino Blando, Qui chi non terrorizza si ammala di terrore, riflette invece su come un "nemico" invisibile come il virus Covid-19, sia stato usato per enfatizzare le differenze e costruire nuove immagini dell'alterità. È il caso dei cinesi, ritenuti responsabili della trasmissione del virus a livello mondiale, tanto che l'allora presidente degli USA Donald Trump, lo aveva più volte definito "chinese virus". L'ultimo saggio, Chi lo sa? Scienza e intellettuali alla prova del Covid-19, di René Capovin si interroga infine sulla questione del sapere, che il virus ci ha imposto di riconsiderare. Il non-sapere certificato di medici e virologi è la messa in mostra di una scienza immaginaria.

Ancora alcune righe, solo per accennare al tema del secondo numero, appena uscito mentre consegno questa recensione. Occulto, segreto, indicibile, a cura di Matteo Aria, è costruito intorno a una categoria ampia ed eterogenea in relazione ai concetti di 'occulto' e di 'segreto', che permette di esplorare i rumors, il patrimonio, le teorie del complotto, l'allarmismo, il terrorismo. Se il nodo teorico del volume precedente era il rapporto natura/cultura, qui, in modo altrettanto forte, si richiama un'altra grande 
dicotomia antropologica: quella tra tradizione e modernità. Gli articoli sono a firma di Pamela J. Stewart (Strathern) e Andrew J. Strathern, Matteo Gallo, Francesco Lattanzi, Mara Benadusi, Giacomo Nerici, Mariano Pavanello, Carolina Kobelinsky, Simona Taliani. 